\section{Neue Pille gegen MS erfolgreich getestet}

Ein neuer oraler Wirkstoff ist jetzt erfolgreich gegen Multiple Sklerose (MS) geprüft worden. Die Substanz mit der vorläufigen Bezeichnung ONO-4641 konnte bis zu $90 \%$ der MS-bedingten Läsionen im Gehirn verhindern.

$\mathrm{D}$ er neue Wirkstoff moduliert Sphingosin-1-Phosphat-Rezeptoren und hält so Lymphozyten davon ab, in das Gehirn zu wandern und dort das Myelin der Nervenfasern anzuknabbern. ONO4641 wirkt damit ähnlich wie das vor kurzem eingeführte orale MS-Therapeutikum Fingolimod.

\section{Zahl der neuen Läsionen reduziert}

In einer Phase-II-Studie hatten Ärzte um Timothy Vollmer von der University of Colorado in Denver die Substanz bei 407 MS-Patienten in drei verschiedenen Dosierungen geprüft. Patienten mit aktiver schubförmiger MS erhielten ein halbes
Jahr lang täglich entweder 50, 100 oder 150 Mikrogramm des Wirkstoffs oder Placebo, teilt die US-Neurologengesellschaft AAN mit. Nach 26 Wochen zeigten die Patienten mit dem neuen MS-Mittel zwischen $77 \%$ und $92 \%$ weniger neue Gadolinium anreichernde Läsionen im MRT als Patienten mit Placebo. Das beste Ergebnis wurde bei den Patienten mit der mittleren Dosierung festgestellt.

Dosisabhängig kam es bei Patienten mit ONO-4641 vermehrt zu unerwünschten Wirkungen wie Bradykardie, Blutdruckveränderungen, $\mathrm{AV}$-Block oder Leberenzymerhöhungen. Eine Lymphopenie wurde bei $4 \%$ der Patienten mit der höchsten

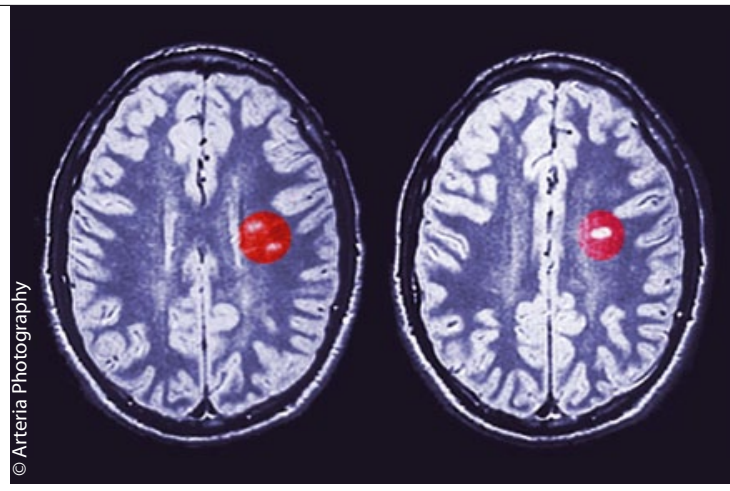

Unter Ono-4641 wiesen die Patienten weniger $\mathrm{Gd}^{+}$-Läsionen auf als unter Placebo.

Dosierung und bei $1 \%$ mit der niedrigsten Dosierung festgestellt.

Die Ergebnisse der Studie werden nun auf der AAN-Jahrestagung (21. bis 28. April 2012) in New Orleans präsentiert. Entwickelt wird das neue Medikament von Merck Serono und Ono Pharmaceuticals.

Thomas Müller

Pressemitteilung der American Academy of Neurology (AAN) vom 10.4.2012

\section{Diabetiker und depressiv: Das lockt Demenzen}

Bis zu $20 \%$ der Typ-2-Diabetiker leiden an einer Depression. Die Gefahr, dass sie auch noch eine Demenz entwickeln, ist deutlich erhöht. In einer großen Registerstudie wurden sie doppelt so oft demenzkrank wie nicht depressive Diabetiker.

S owohl eine Depression als auch ein Typ-2-Diabetes haben sich in früheren Studien bereits als unabhängige Risikofaktoren für eine Demenz entpuppt. In der Diabetes and Aging Study wurde jetzt untersucht, wie die kognitive Leistungsfähigkeit durch das Zusammentreffen dieser Risikofaktoren beeinflusst wird.

Von insgesamt 19.239 Typ-2-Diabetikern wurden bei Studienbeginn 3.766 $(19,6 \%)$ - basierend auf einer ICD-9Diagnose und/oder einer antidepressiven Therapie in den zwölf vorausgegangenen Monaten - als depressiv eingestuft. Um auszuschließen, dass es sich bei einer Depression um das Frühsymptom einer Demenz handelte, wurden für die Analyse nur Demenzerkrankungen berücksichtigt, die drei bis fünf Jahre später festgestellt wurden. Eine Demenzdiagnose gemäß ICD-9-CM (Altersdemenz, Alzheimer-Demenz, vaskuläre Demenz oder nicht genauer spezifizierte Demenz) erhielten in diesem Zeitfenster 2,1 \% der
Patienten mit und 1,0\% der Patienten ohne Depression.

\section{Es liegt nicht (nur) am ungesunden Lebensstil}

Die depressiven Diabetespatienten nahmen es mit Ernährungsempfehlungen und Therapievorschriften nicht so genau, hatten dementsprechend einen höheren BMI und eine schlechtere Stoffwechselkontrolle sowie mehr Begleiterkrankungen und waren auch öfter Raucher. Obwohl all diese Faktoren pathologische Gefäßveränderungen und die Entstehung einer Demenz fördern, konnten sie den beobachteten Zusammenhang zwischen Depression und Demenz nicht erklären. Auch wenn ihr Einfluss herausgerechnet wurde, ergab sich bei den depressiven Diabetikern immer noch ein um $100 \%$ erhöhtes Demenzrisiko.

Verantwortlich dafür sind, so die Studienautoren um Wayne Katon von der University of Washington School of Me- dicine in Seattle, vermutlich biologische Faktoren, die mit der Depression in $\mathrm{Zu}$ sammenhang stehen. So könnte zum Beispiel die bei depressiven Patienten vermehrte Kortisolproduktion Schäden im Hypothalamus anrichten und auch die Neurogenese beeinträchtigen. Durch die erhöhten Kortisolspiegel wird außerdem das metabolische Syndrom verstärkt, das ebenfalls als Risikofaktor für Alzheimer und vaskuläre Demenzen gilt. Des Weiteren könnten die verstärkte Freisetzung proinflammatorischer Faktoren und eine erhöhte Insulinresistenz zum geistigen Abbau beitragen.

\section{Demenzprävention mit Antidepressiva?}

Laut Kane et al. deuten die Studienergebnisse darauf hin, dass das Demenzrisiko durch das gemeinsame Auftreten von Diabetes und Depression noch stärker erhöht wird, als durch eine der Erkrankungen allein. Jetzt gelte es zu prüfen, ob durch die Behandlung einer Depression auch das Demenzrisiko gesenkt werden könne.

Dr. Beate Schumacher

Katon W et al. Arch Gen Psychiatry. 2012; 69 (4): 410-17. doi:10.1001/archgenpsychiatry.2011.154 